\title{
Uniendo las piezas del puzzle: a propósito de un caso de trastorno del espectro autista adulto.
}

Fitting the puzzle pieces: a case report of adult autism spectrum disorder.

Cynthia Binellia, Gemma Caupena ${ }^{\mathrm{b}}$, Carla Pedreño ${ }^{\mathrm{c}}$.

${ }^{a}$ Residente de Psicología Clínica (PIR 4). Corporació Sanitària Parc Taulí. Sabadell, Barcelona, España. ${ }^{b}$ Psiquiatra. Salud Mental Adultos. Corporació Sanitària Parc Taulí. Sabadell, Barcelona, España.

${ }^{c}$ Psicóloga. Departamento de Psicología Clínica y de la Salud. Universidad Autónoma Barcelona, Bellaterra, España.

Correspondencia: Cynthia Binelli (cynthiabinelli@gmail.com)

Recibido: 30/12/2012; aceptado: 20/05/2013

RESUMEN: Los trastornos del espectro autista representan uno de los retos más importantes de la psiquiatría actual. A pesar de los importantes avances diagnósticos y de tratamiento para esta población en edad infanto-juvenil, existe escasa literatura sobre la evolución y el tratamiento de estos pacientes en la etapa adulta.

PALABRAS CLAVE: trastorno autístico; adulto; terapia; evolución clínica.
ABSTRACT:Autism spectrum disorders are one of the most important challenges of current psychiatry. Despite the significant progress on diagnosis and treatment of these patients during childhood stages, little is known about the course and treatment of these patients during adulthood. KEY WORDS: autistic disorder; adult; therapy; clinical evolution.

\section{Caso Clínico}

Se trata de una paciente de 18 años, diagnosticada (DSM-IV-TR) de Fobia social y Trastorno dependiente de la personalidad, derivada desde el Servicio de Salud Mental Infanto-Juvenil (CSMIJ) para seguimiento en el servicio de adultos, que acude acompañada por su madre.

Es la mayor de dos hermanas. Padres separados. Vive con la madre y la hermana. Durante su infancia destaca déficit de atención y mal rendimiento académico sin repetir curso. En el momento actual comienza a cursar un módulo superior y colabora en el negocio familiar. Como antecedentes médicos de interés, destaca un retraso en el crecimiento aislado que requirió tratamiento con hormona del crecimiento $(\mathrm{GH})$ de los 12 a los 17 años. Intolerancia a la fructosa asociada a sangrados digestivos e intolerancia a la lactosa. En la infancia se realiza examen genético, sin observarse ninguna alteración genética, ni numérica ni estructural. Niega consumo de tóxicos. Entre los antecedentes psiquiátricos familiares la madre refiere sintomatología ansio- 
sa de larga evolución. Describen al padre como una persona solitaria, introvertida, y "poco emocional", características que presentan varios familiares por línea paterna. Desde el punto de vista psiquiátrico, la paciente entró en contacto con el CSMIJ a los 14 años, siendo la orientación diagnóstica de Fobia Social. A los 17 años ingresó en Hospital de Día durante 2 meses por dificultades para relacionarse, absentismo escolar y gran dependencia del ámbito familiar. La orientación diagnóstica fue de Fobia Social y Trastorno Dependiente de Personalidad (según evolución) que tras el alta evolucionó hacia una mejoría parcial. Entre los rasgos de personalidad premórbidos destaca elevada introversión. Su madre la describe como una niña aislada, "diferente", con escaso interés social.

De la exploración inicial destaca un contacto distante, lenguaje poco espontáneo sin alteraciones a nivel formal. Ansiedad psico-física moderada, ideas obsesivas de tipo hipocondríaco, onicofagia y bruxismo nocturno. No se objetivan síntomas de la esfera afectiva ni psicótica. No alteraciones del curso ni contenido del pensamiento. No alteraciónes de la sensopercepción. Consciencia parcial trastorno.

El cuadro se orienta como un Trastorno de la Personalidad Cluster C (rasgos fóbicos, obsesivos y dependientes). Se mantiene tratamiento farmacológico pautado desde CSMIJ (Sertralina, $100 \mathrm{mg}$ ), y se deriva a psicología para trabajar dificultades sociales. Realiza pocas visitas con mal cumplimiento de las estrategias propuestas (modelo cognitivo-conductual), y se desvincula por incompatibilidad con estudios.

\section{Segundo contacto al cabo de dos años}

La paciente se re-vincula tras casi dos años (20 años) de no realizar seguimiento. Persisten dificultades sociales. Se realizan visitas de exploración por parte de psiquiatría y psicología, incluida una visita con la madre. Expresan la decisión de abandonar medicación ISRS. No aceptan tratamiento farmacológico, aunque si tratamiento psicológico. Durante la valoración, la paciente impresiona de un contacto "peculiar" más que del contacto fóbico-ansioso habitual. Presenta una extraña -aunque sutil- alteración en el contacto ocular. El lenguaje continúa siendo poco espontáneo. Además de las dificultades a nivel social y el escaso interés por el entorno, destacan ideas obsesivas de tipo hipocondríaco y un rechazo absoluto por el contacto físico y sexual. Con el objetivo de valorar las dificultades/habilidades de la paciente a nivel interpersonal, se plantean ejercicios de exposición social simples fuera de consulta. La paciente no realiza ninguno de los ejercicios/estrategias propuestas. Tampoco destaca preocupación por resolver estas dificultades. Por el contrario, impresiona de cierta indiferencia al respecto. Ante ello, comienzan las dudas respecto a la evitación social (¿incómoda vs. ansiosa?), y en cuanto al deseo de relacionarse socialmente, (¿discurso social aprendi- 
do?). Ante estas dudas surge la sospecha de que estos síntomas pudieran explicarse por otros diagnósticos: ¿rasgos esquizoides? ¿rasgos del espectro autista?; por lo que se plantea una nueva evaluación de la paciente.

Protocolo de evaluación y resultados de los mismos:

- Entrevista Clínica Estructurada para los Trastornos del Eje II del DSM-IV (SCID II):

- Criterios Trastorno Esquizoide de la Personalidad: 4/5. Próxima a punto de corte.

- Criterios Trastorno Evitativo de la Personalidad: 5/5. Cumple punto de corte.

- Pruebas de Teoría de la Mente (TOM):

- "Test Historias de Happé": Muestra una capacidad adecuada para inferir estados mentales en otros, entender metáforas y dobles sentidos.

- "Test de lectura de la Mente en los Ojos": Es capaz de atribuir a la mayoría de elementos (23/36) el sentimiento o emoción adecuado que muestra la fotografía.

- "Test de las Meteduras de Pata": Identifica correctamente las historias de "metedura de pata" pero muestra dificultades en atribuir estados emocionales e intencionalidad a los personajes.

- Escala de Inteligencia Wechsler para Adultos (WAIS-III):

CI Verbal $=99$ CI Manipulativo $=94$ CI Total $=97$ Rango: Medio

- Entrevista para el diagnóstico del autismo revisada (ADI-R): Se identifican alteraciones en la interacción social recíproca. Limitada variedad de expresiones faciales para comunicarse y regular la interacción social. Incapacidad para desarrollar relaciones con iguales. Se objetiva una falta de reciprocidad socio-emocional, respuestas sociales inadecuadas y un déficit en la búsqueda de compartir placer con los otros. Retrospectivamente se identifican alteraciones cualitativas de la comunicación, como falta de juego simbólico espontáneo y variado.

También se identifican algunos patrones de conducta restringidos.

- Resonancia Magnética (RM): Examen craneal dentro de la normalidad.

\section{Valoración Global:}

A lo largo de la exploración la paciente se muestra colaboradora aunque poco motivada. La información obtenida permite objetivar signos de un trastorno del espectro autista (TEA) en el que destaca escaso interés social, expresiones y conductas 
inapropiadas, dificultades para establecer relaciones sociales con iguales, expresiones faciales y afectividad restringida, e intereses/actividades limitadas. El perfil cognitivo y la ejecución en los dominios "TOM" (número de errores no-significativo, aunque dificultad en la atribución de estados emocionales e intencionalidad a los personajes en el test "Meteduras de Pata") son coherentes al perfil observado en pacientes TEA de alto funcionamiento (1).

\section{Evolución}

Se realizó la devolución a la paciente y su familia, proporcionando información sobre el diagnóstico. Ello desculpabilizó y tranquilizó a la familia, pudiendo dar una explicación a actitudes y conductas "poco entendibles" con las que convivieron muchos años. Así, respuestas emocionales incongruentes a la situación, el fracaso de los intentos por parte de la familia para que la paciente se socializara, la gran dependencia del ámbito familiar, y aquella actitud aparentemente pasiva hacia el entorno pudieron entenderse en el contexto de un trastorno que daba coherencia a muchos años de "no entender". A nivel farmacológico se planteó instaurar un fármaco antipsicótico atípico a dosis bajas, pero la paciente lo rechazó. A nivel psicológico el trabajo se orientó en un primer nivel, a realizar una psicoeducación del trastorno. Se trabajaron dificultades asociadas a las prácticas de empresa que la paciente estaba realizando, principalmente asociadas a respuestas inadecuadas por falta de entendimiento de las claves sociales. La paciente finalizó el módulo y sigue colaborando en el negocio familiar. Continúa realizando seguimiento individual orientado a trabajar las dificultades del trastorno, y será incluida en un grupo para pacientes con diagnóstico TEA que próximamente realizaremos en el servicio.

\section{Discusión}

Los trastornos del espectro autista representan uno de los retos más importantes de la psiquiatría actual. Se trata de un grupo de trastornos del neurodesarrollo englobados en el apartado de trastornos generalizados del desarrollo según la clasificación del Manual Diagnóstico y Estadístico para trastornos mentales (DSM-IV TR) (2), y que incluye el Trastorno Autista, Trastorno de Asperger, Trastorno de Rett, Trastorno Desintegrativo Infantil, y el Trastorno Generalizado del Desarrollo No Especificado. Aunque los diagnósticos incluyen diferencias en la presentación clínica, existen tres grandes núcleos afectados que comparten estos trastornos: la alteración cualitativa 
de la interacción social, la alteración cualitativa de la comunicación, y un patrón de comportamientos, intereses y/o actividades restrictivos, repetitivos y estereotipados. La presentación clínica y el curso evolutivo son heterogéneos. Ello ha impulsado a que la literatura haga referencia a sujetos de "alto o bajo funcionamiento" en función del grado de afectación y funcionalidad. En este sentido, las propuestas para próxima versión del DSM (DSM 5), contemplan incluir un sistema de clasificación dimensional, en el que se unifican todos los diagnósticos a un único diagnóstico de TEA en función del nivel de afectación y el grado de soporte necesario para funcionar (3).

El importante incremento en la prevalencia de este grupo de trastornos debe contextualizarse en el recorrido y evolución que han tenido los TEA en la historia de la psiquiatría. Mientras que en los años 60, -previo a la implementación del sistema de clasificación psiquiátrica-, se estimaba en Europa una prevalencia de 4.1/10,000 hab., en la década del 2000 ya se había incrementado a una media de 18,75/10,000 hab. (4), con mayor afectación en hombres que mujeres. Datos similares se observan en población adulta en Reino Unido (5). No se disponen de datos epidemiológicos en población española.

Al menos un $10 \%$ de pacientes del espectro están asociados a una alteración cromosómica o genética. Entre un 11 y $40 \%$ desarrollan epilepsia, con una incidencia más elevada de ciertas alteraciones de salud que la población general (6). A nivel psiquiátrico existe importante comorbilidad con los trastornos de ansiedad, el trastorno obsesivo compulsivo, los trastornos del sueño, el trastorno por déficit de atención y la depresión. Aproximadamente un 50\% presenta un déficit cognitivo asociado (7-8).

La evolución es heterogénea y está asociada al nivel de gravedad. Dentro de los principales factores asociados al pronóstico se encuentran el nivel intelectual, el desarrollo temprano del lenguaje, la severidad de los síntomas, y el diagnóstico e intervención precoz (9-10). En este sentido, el importante avance realizado en los últimos años en la detección y la intervención temprana han cambiado de forma radical el escenario de estos pacientes. Actualmente, son las intervenciones conductuales y los grupos psicoeducativos para padres los tratamientos que han demostrado eficacia en población infantil, aunque también se recomienda el uso de promoción de competencias sociales, sistemas alternativos de comunicación, terapia cognitivoconductual, y el sistema de tratamiento y educación para niños con autismo y problemas de comunicación relacionados ("TEACCH") (11). A nivel farmacológico, dos antipsicóticos atípicos, -la risperidona y el aripiprazol-, han demostrado eficacia en el tratamiento de la irritabilidad, las conductas agresivas y conductas autolesivas asociadas al trastorno (12-13). En caso de comorbilidad con trastorno depresivo, trastorno de ansiedad y conductas obsesivas está indicado el uso inhibidores selectivos de la recaptación de serotonina (ISRS) (14-16) así como de metilfenidato y la atomoxetina en comorbilidad con trastorno por déficit de atención con hiperactividad (17-18). Un aspecto de interés en nuestra paciente gira entorno al tratamiento con GH que realizó 
durante 5 años para el retraso en el crecimiento. El factor de crecimiento insulínico tipo1 (IGF-1), una proteína cuya producción es estimulada por la GH, es uno de los tratamientos aprobados por la FDA en EEUU para el retraso en el crecimiento. Desde el Instituto Tecnológico de Massachusetts (MIT) se realizó un estudio que observó un efecto corrector en el proceso de maduración sináptica tras administrar IGF-1 a ratones portadores del gen afectado en el Síndrome de Rett (RTT), un trastorno asociado al autismo (19). Estos hallazgos han sido replicados en una muestra de seis pacientes afectados del RTT, sin observarse riesgos asociados a la administración del tratamiento (20). Aunque estos hallazgos deben ser adecuadamente replicados en muestras más amplias, los autores proponen la administración de IGF-1 en el tratamiento de este síndrome y su potencialidad en trastornos asociados a retrasos de la maduración sináptica. En esta línea, el "Seaver Autism Center" del Hospital Mount Sinai en Nueva York ha iniciado recientemente el primer ensayo clínico en población autista utilizando IGF-1, con el objetivo de evaluar la eficacia, tolerancia, y seguridad en el tratamiento de uno de los principales ejes del trastorno: los déficits sociales (21). Los importantes esfuerzos y avances científicos resolverán algunas de estas dudas en los próximos años y contribuirán a esclarecer la eficacia y la potencialidad de estas terapias emergentes. En nuestro caso, queda abierto el interrogante sobre el efecto indirecto de la terapia hormonal realizada por la paciente los síntomas del trastorno.

En relación a la evolución de estos trastornos en la vida adulta, existe escasa literatura sobre la evolución y el tratamiento en esta etapa. Estudios en población adulta sugieren que tan solo una minoría vive de forma independiente, trabaja, estudia a tiempo completo, se casa o tiene pareja estable, y que incluso en sujetos de alto funcionamiento, la funcionalidad y la adaptación está por debajo de la capacidad intelectual (22-24). A pesar de que la detección e intervención temprana suponen una mejora evidente en el pronóstico de estos pacientes, al tratarse de un diagnóstico estable, persisten dificultades en la edad adulta, sumada a las comorbilidades psiquiátricas asociadas. Otro aspecto de vital importancia es la transición de estos sujetos a la vida adulta (25), donde las propias limitaciones del trastorno afectan áreas vitales en este período. A ello, se suma la transición de los servicios de salud mental infantil donde generalmente han realizado un tratamiento estructurado e intensivo, a un seguimiento menos estructurado en los servicios de salud mental para adultos, donde es poco habitual disponer de programas específicos para dicho trastorno. Paralelamente, los servicios de salud mental de adultos, por un lado, comienzan a asumir el seguimiento de todos estos casos que hoy en día, -afortunadamente- están siendo diagnosticados de forma precoz, y por otro, comienzan a detectar nuevos casos no diagnosticados en la infancia, lo que generalmente ocurre en sujetos de alto funcionamiento. Este importante incremento en la prevalencia y la falta de tratamientos y dispositivos adecuados para población autista adulta, llevaron a que en el año 2010 un grupo de expertos de EEUU se reuniera para reflexionar sobre las necesidades de 
estos sujetos en esta etapa (25). Considerando la prevalencia actual en dicho país, y teniendo en cuenta que la esperanza de vida de estos pacientes es en su mayoría similar a la población general, se estimó que en los próximos 20 años habrá en EEUU 200,000 casos de personas con un TEA <65. Ello supone no sólo un importante reto clínico, sino la necesidad de establecer programas adecuados a esta población. En este contexto, desde hace algunos años el Servicio de salud mental infanto-juvenil de nuestro hospital cuenta con un programa pionero en TEA. Para dar continuidad a la labor que se está realizando desde la unidad infanto-juvenil y para dar respuesta a todos aquellos casos que realizan seguimiento en la unidad de adultos, ha surgido la iniciativa de realizar una terapia de grupo para personas con TEA en etapa adulta, que actualmente se encuentra en fase de implementación. No debemos olvidar que la etapa adulta va unida a retos vitales como la pareja, la sexualidad, la vida laboral/ social, y la independencia del ámbito familiar, que pueden resultar especialmente complicadas para estos sujetos.

El recorrido que han realizado los TEA dentro de la psiquiatría en las últimas tres décadas han posicionado a este grupo de trastornos y a los clínicos en un escenario mucho más definido, delimitando los criterios diagnósticos, estableciendo herramientas adecuadas para el diagnóstico y la detección precoz, creando una serie de tratamientos siguiendo criterios de eficacia, y estableciendo programas y recursos específicos para población infanto-juvenil. Queda mucho camino por recorrer. Y dentro de este camino está, sin duda, el reto de los trastornos del espectro autista en la etapa adulta.

\section{Conclusiones}

A pesar de que inicialmente los diferentes síntomas del caso presentado (dificultades en las relaciones sociales, rasgos obsesivos, sintomatología fóbica, elevada dependencia..) eran coherentes con un diagnóstico de un trastorno de la personalidad cluster C, la persistencia de los síntomas y la evolución tórpida, la poca respuesta a los diferentes tratamientos, sumados a un contacto distante, al contacto ocular peculiar, la afectividad restringida, e intereses/actividades limitadas, pudieron entenderse desde un enfoque evolutivo por un trastorno del espectro autista. Incluso, otros síntomas presentes desde la infancia y aparentemente no relacionados, como son las alteraciones gastrointestinales, el déficit de hormona del crecimiento, la onicofagia, las alteraciones del sueño y el déficit de atención -todos ellos más frecuentes en esta población-, ahora, todos unidos, acaban de dar forma al puzzle. 


\section{BIBLIOGRAFÍA:}

(1) Spek AA, Scholte EM, Van Berckelaer-Onnes IA. Theory of mind in adults with HFA and Asperger syndrome. J Autism Dev Disord. 2010;40(3):280-9.

(2) American Psychiatric Association. Diagnostic and statistical manual of mental disorders. Fourth ed. Washington, D.C: American Psychiatry Asociation: 1994.

(3) Tanguay PE. Autism in DSM-5. Am J Psychiatry. 2011;168(11):1142-4.

(4) Elsabbagh M, Divan G, Koh YJ, Kim YS, Kauchali S, Marcín C, el al. Global prevalence of autism and other pervasive developmental disorders. Autism Res. 2012;5(3):160-79.

(5) Brugha TS, McManus S, Bankart J, Scott F, Purdon S, Smith J, et al. Epidemiology of Autism Spectrum Disorders in Adults in the Community in England. Arch. Gen Psychiatry. 2011;68(5):459-65.

(6) Spence SJ, Schneider MT. The role of epilepsy and epileptiform EEGs in autism spectrum disorders. Pediatr Res. 2009;65:599-606.

(7) Skokauskas N, Gallagher L. Psychosis, affective disorders and anxiety and autistic spectrum disorder: Prevalence and nosological considerations. Psychopathology. 2010;43:8-16.

(8) Krakowiak P, Goodlin-Jones B, Hertz-Picciotto I, Croen LA, Hansen RL. Sleep problems inchildren with autism spectrum disorders, developmental delays, and typical development: A populationbased study. J Sleep Res. 2008;17:197-206.

(9) McGovern CW, Sigman M. Continuity and change from early childhood to adolescence in autism. J Child Psychol Psychiatry. 2005;46:401-08.

(10) Turner LM, Stone WL, Pozdol SL, Coonrod EE. Follow-up of children with autism spectrum disorders from age 2 to age 9. Autism. 2006;10:243-64.

(11) Fuentes-Biggi J, Ferrari-Arroyo MJ, Boada-Muñoz L, Touriño-Aguilera E, Artigas-Pallarés J, Belinchón-Carmona M, et al. Guía de buena práctica para el tratamiento de los trastornos del espectro autista. Rev Neurol. 2006; 43(7):425-38.

(12) McCracken JT, McGough J, Shah B, Cronin P, Hong D, Aman MG, et al. Risperidone in children with autism and serious behavioral problems. N Engl J Med. 2002;347(5):314-21.

(13) Owen R, Sikich L, Marcus RN, Corey-Lisle P, Manos G, McQuade RD, et al. Aripiprazole in the treatment of irritability in children and adolescents with autistic disorder. Pediatrics. 2009;124(6):153340 .

(14) Hollander E, Phillips A, Chaplin W, Zagursky K, Novotny S, Wasserman S, et al. A placebo controlled crossover trial of liquid fluoxetine on repetitive behaviors in childhood and adolescent autism. Neuropsychopharmacology. 2005;30(3):582-9.

(15) Steingard RJ, Zimnitzky B, DeMaso DR, Bauman ML, Bucci JP. Sertraline treatment of transition-associated anxiety and agitation in children with autistic disorder. J Child Adolesc Psychopharmacol. 1997;7(1):9-15.

(16) Martin A, Koenig K, Anderson GM, Scahill L. Low-dose fluvoxamine treatment of children and adolescents with pervasive developmental disorders: a prospective, open-label study. J Autism Dev Disord. 2003;33(1):77-85.

(17) Research Units on Pediatric Psychopharmacology Autism Network. Randomized, controlled, crossover trial of methylphenidate in pervasive developmental disorders with hyperactivity. Arch Gen Psychiatry. 2005;62(11):1266-74. 

NOTAS CLÍNICAS

(18) Arnold LE, Aman MG, Cook AM, Witwer AN, Hall KL, Thompson S, et al. Atomoxetine for hyperactivity in autism spectrum disorders: placebo-controlled crossover pilot trial. J Am Acad Child Adolesc Psychiatry. 2006;45(10):1196-205.

(19) Tropea D, Giacometti E, Wilson NR, Beard C, McCyrry C, Fu DD, et al. Partial reversal of Rett Syndrome-like symtoms in MeCP2 mutant mice. Proc Natl Acad Sci USA. 2009; 106(6):2029-34.

(20) Pini G, Scusa MF, Congiu L, Benincasa A, Morescalchi P, Bottiglioni I, et al. (in press). IGF1 as a potential treatment for Rett Syndrome: Safety assesment in six Rett Patients. Autism Res Treat.

(21) Mount Sinai School of Medicine: Seaver Autism Center (consultado el 23-12-2012). Disponible en http://www.mssm.edu/research/centers/seaver-autism-center/research/current-studies

(22) Howlin P. Outcome in adult life for more able individuals with autism or Asperger syndrome. Autism 2000;4:63-83.

(23) Orsmond GI, Krauss MW, Seltzer MM. Peer relationships and social and recreational activities among adolescents and adults with autism. J Autism Dev Disord 2004;34:245-56

(24) Taylor JL, Seltzer MM. Employment and post-secondary educational activities for young adults with autism spectrum disorders during the transition to adulthood. J Autism Dev Disord 2011;41: 566-74.

(25) Piven J, Rabins P. Autism spectrum disorders in older adults: toward defining a research agenda. J Am Geriatr Soc 2011;59(11):2151-55. 